\title{
Clinical Efficacy of Fosfomycin for the Treatment of Complicated Lower Tract and Uncomplicated Urinary Tract Infections

\author{
Jerod L. Nagel ${ }^{1,3}$, Laraine Washer ${ }^{2}$, Anjly Kunapuli ${ }^{1,3}$, \\ Jennifer Heidmann ${ }^{3}$, Jennifer Pisani ${ }^{3}$, Tejal Gandhi'
}

\section{Abstract}

Fosfomycin is an oral antibiotic with activity against multidrug resistant organisms, including vancomycin-resistant enterococcus (VRE) and extended-spectrum $\beta$-lactamase (ESBL) producing Enterobacteriaceae. However, there is currently no information describing efficacy of fosfomycin compared to other agents for healthcare associated UTIs. Additionally, there is minimal information characterizing the potential cost savings with utilization of fosfomcyin versus traditional therapies. This retrospective study evaluated clinical and economic outcomes of fosfomycin compared to matched controls. The controls were before the addition of fosfomycin to hospital formulary with recommended prescribing criteria. The fosfomycin group consisted of patients who were admitted to the hospital after the addition of fosfomycin to the hospital formulary with recommended prescribing criteria. Patients receiving fosfomycin for the treatment of UTI were matched to control patients based on pathogen, renal function, and presence of a lower UTI. A total of 86 patients were evaluated. The majority of patients received fosfomycin for the treatment of VRE (45.6\%) and ESBL producing Enterbacteriaceae (16.3\%) UTIs. Patients with a combination of allergies or documented resistance to first line agents also received fosfomycin to treat Enterococcus (25.6\%), Enterobacteriaceae (7.8\%), or polymicrobial UTIs (4.7\%). Doxycycline, nitrofurantoin, sulfamethoxazole/trimethoprim, meropenem, and linezolid were the most common antibiotics prescribed in the control group. The average days of treatment were lower in the fosfomycin group (2.93 vs. 7.19 days, $p<0.0001)$. Fosfomcyin was associated with similar clinical success rates (95 vs. $95 \%, p>0.99)$ and recurrence rates (4.7 vs. $4.7 \%, p>0.99$ ).
1 University of Michigan Hospitals and Health System, Department of Pharmacy, Ann Arbor, Michigan, USA.

2 University of Michigan Hospitals and Health System, Division of Infectious Diseases, Ann Arbor, Michigan, USA.

3 University of Michigan College of Pharmacy, Ann Arbor, Michigan, USA.

Contact information:

\section{Jerod L. Nagel.}

Running Head: Fosfomycin for Treatment of Urinary Tract Infections.

Address: 1111 E Catherine Street, Rm 300. Ann Arbor, MI 48109.

झ nagelj|@med.umich.edu

Keywords

UTI, Fosfomycin, VRE, ESBL. 
Additionally, the mean antibiotic cost per patient was lower in the fosfomycin group (\$106.74 vs. \$269.55). Adding fosfomycin to formulary resulted in similar clinical success rates and lower cost for the treatment of complicated lower and uncomplicated UTIs.

\section{Background}

In recent years, multi-drug resistant (MDR) urinary tract infections (UTI) caused by extended spectrum $\beta$-lactamase (ESBL) producing Enterobacteriaceae and vancomycin-resistant Enterococci (VRE) have become more prevalent. [1-3] Due to high resistance rates to oral antibiotics, VRE and ESBL UTIs are commonly treated with intravenous antibiotics which are more expensive and inconvenient for patients. $[2,4]$ Thus, there is a need for less expensive and effective alternative antibiotics for the treatment of VRE and ESBL UTIs. [5-9] Fosfomycin tromethamine is a synthetic, broad spectrum, bactericidal antibiotic that works by inhibiting pyruvyl transferase during bacterial cell wall synthesis, and it may also decreases bacterial adherence to epithelial cells in the urinary tract. [10] Fosfomycin is available as an orally administered single-dose 3 gram sachet that is mixed with water. [10] Some reports utilize 3-doses of fosfomycin for the treatment of complicated lowertract UTIs, but efficacy and safety of this regimen has never been compared to single-dose regimens. [11] Fosfomycin has demonstrated efficacy in both in vitro and in vivo studies for treating UTIs caused by ESBL-producing Enterobacteriaceae, [12-15] while in vitro studies have shown high susceptibility of VRE to fosfomycin with susceptibilities of $98 \%$. [5-7] Clinical data are limited to bolster the use of fosfomycin; however, a recent study showed high microbiological cure rates and in vitro susceptibility of VRE and ESBL UTI isolates to fosfomycin. [16] In 2010, the University of Michigan Health System
(UMHS) Antimicrobial Stewardship Program introduced a guideline recommending the use of fosfomycin tromethamine for the treatment of lower UTIs caused by VRE or ESBL producing organisms that are resistant to other oral antibiotics.

\section{Objectives}

The aim of this study was to evaluate the clinical efficacy of fosfomycin for the definitive treatment of VRE and ESBL lower tract UTIs in addition to those UTIs caused by non-VRE Enterococci and non-ESBL Enterobacteriaceae that are unable to be treated with oral antibiotics due to patient allergies or resistance. This study also determined cost savings to the institution following implementation of the guideline.

\section{Methods}

This retrospective matched cohort study was conducted at the University of Michigan Health System, a 930-bed academic hospital, and received Institutional Review Board approval. Clinical outcomes were evaluated for patients who received fosfomycin from January 1, 2011 to December 31, 2011, and compared with a matched control group who received alternative treatment regimens during 2009-2010. The fosfomycin group included all patients who received a dose of fosfomycin in the year 2011 after implementation of an institutional guideline which provided treatment recommendations for uncomplicated and complicated lower UTI. This guideline recommended the use of fosfomycin 
tromethamine for the treatment of lower UTIs caused by VRE or ESBL producing organisms that are resistant to other oral antibiotics. The patients in the fosfomycin group were matched to historical controls from 2009 and 2010 based on organism and creatinine clearance $(\mathrm{CrCl})$ greater than $50 \mathrm{ml} / \mathrm{min}$. Patients were considered to have a UTI if patient had documented UTI-specific symptoms (dysuria, frequency or urgency) in combination with elevated white blood cells per high powered field on urinalysis. Patients were also considered to have a UTI if the patient had a condition which made UTI specific symptoms undetectable (presence of urinary catheter, altered mental status, quadrapalegic). This study defined UTI as the following: physician diagnosed patient as having a UTI, presence of documented symptoms or abnormal urinalysis, and the patient received treatment with antibiotics. Inclusion criteria consist of patients greater than or equal to 18 years old who received treatment for a complicated or uncomplicated lower UTI caused by Enterococcus or Enterobacteriacae. Patients were excluded if pregnant, or recent renal transplant.

UTI symptoms defined as dysuria or increased urgency or frequency of urination were collected when available. Urinalysis was analyzed for white blood cell count greater than 10 per high powered feild, positive leukocyte esterase, and positive nitrite. Urine culture results were collected including the organism isolated, number of organisms (CFU/ml), susceptibility to fosfomycin, and the date of culture. All organisms identified in the urine culture were collected. Routine fosfomycin susceptibility testing was performed for all VRE isolates from urine using Kirby Bauer and upon request for other organisms. Urine culture results with the same organism were documented for all repeat positive cultures within 30 days of the original culture. Any repeat cultures were further investigated to determine whether colonization or infection were present. In the fosfomycin group, antibiotics received within 2 days of fosfomycin therapy were recorded. In the control group, all antibiotics used to treat the UTI were recorded. Antibiotic data collected included dose, frequency, duration, and total grams administered to the patient. Dosing recommendations for fosfomycin in the UMHS guidelines include 3-gram single dose therapy for uncomplicated UTIs and 1-3 doses every 48 hours for complicated UTIs.

The primary endpoint was clinical success of UTI treatment, which was defined as having a urine culture within 30 days of the original culture that was not positive for the same organism. As this was a retrospective study, patients were not required to have a repeat urine culture. The secondary endpoint for the study was the total cost of antibiotic therapy for each patient for the treatment of the UTI using the total amount of antibiotic in grams received by the patient. The average wholesale price (AWP) of each medication was used in the cost analysis. Continuous variables were compared using the Student $T$ test and categorical variables were evaluated using Chi-square and Fisher's exact test.

\section{Results}

A total of 86 patients were evaluated which included 43 patients in the fosfomycin group and 43 patients in the control group. Both patient demographics and UTI characteristics were generally similar between the fosfomycin and control groups (Table 1). Patients in the control group had significantly higher $\mathrm{CrCl}$ (79 vs. $58 \mathrm{ml} / \mathrm{min}, P<0.001)$. Treatment of resistant pathogens made up the largest proportion of fosfomycin use with $45 \%$ of patients receiving treatment for $\mathrm{VRE}$ and $16 \%$ for ESBLproducing Enterbacteriaceae UTIs. Due to a combination of allergies, resistance, or contraindications to commonly prescribed oral antibiotics, the remaining patients received treatment for non-VRE Enterococcus (25\%), non-ESBL Enterbacteriaceae (8\%), and polymicrobial (5\%) UTIs. The most common dosing regimen for fosfomycin received by $81.4 \%$ of patients (35/43) was 3 grams orally for one dose. 
Table 1. Patient Characteristics.

\begin{tabular}{|l|c|c|c|}
\multicolumn{1}{|c|}{$\begin{array}{c}\text { Independent } \\
\text { variables }\end{array}$} & $\begin{array}{c}\text { Fosfomycin } \\
\text { N=43 }\end{array}$ & $\begin{array}{c}\text { Control } \\
\text { N=43 }\end{array}$ & p-value \\
\hline Demographics & 63.2 & 60.2 & 0.44 \\
\hline Age & $35(81 \%)$ & $28(65 \%)$ & 0.14 \\
\hline $\begin{array}{l}\text { Female } \\
\text { Creatinine clearance } \\
\text { (ml/min) }\end{array}$ & 58 & 79 & $<0.001$ \\
\hline $\begin{array}{l}\text { UTI Characteristics } \\
\text { Complicated lower } \\
\text { tract UTI }\end{array}$ & $34(79 \%)$ & $41(95 \%)$ & 0.049 \\
\hline $\begin{array}{l}\text { Documented UTI } \\
\text { symptoms }\end{array}$ & $29(67 \%)$ & $24(56 \%)$ & 0.38 \\
\hline $\begin{array}{l}\text { WBC > 10 in UA } \\
\text { Leukocyte esterase }\end{array}$ & $25(68 \%)$ & $25(68 \%)$ & $>0.99$ \\
\hline positive & $32(74 \%)$ & $29(67 \%)$ & 0.48 \\
\hline Nitrate positive & $5(12 \%)$ & $5(12 \%)$ & $>0.99$ \\
\hline
\end{tabular}

The remaining 19\% (8/43) received a variety of multiple dose regimens with 3 grams every 48 hours for three doses being the most common regimen. Nitrofurantoin, sulfamethoxazole/trimethoprim, and doxycycline were the most utilized antibiotics in the control group (Table 2). The average days of treatment were lower in the fosfomycin group (2.93 vs. 7.19 days, $p<0.0001)$.

The clinical success rate was identical in both the fosfomycin and control groups with 95\% of patients (41/43) not having a repeat positive culture of the same organism within 30 days. Both groups also had 2 patients (5\%) with repeat positive cultures and hospital readmission for UTI within 30 days. One patient from the fosfomycin group had a repeat positive culture for VRE faecium, and the other patient had a repeat urine culture positive for ESBL Escherichia coli and Klebsiella pneumoniae. In the control group, one patient had a repeat urine cul-

Table 2. Antibiotic utilization including both empiric and definitive UTI therapy.

\begin{tabular}{|l|c|c|c|c|}
\hline \multicolumn{1}{|c|}{ Antibiotic } & $\begin{array}{c}\text { Antibiotic } \\
\text { Utilization } \\
\text { Fosfomycin Group }\end{array}$ & $\begin{array}{c}\text { Antibiotic } \\
\text { Utilization } \\
\text { Control Group }\end{array}$ & $\begin{array}{c}\text { Antibiotic Days } \\
\text { Fosfomycin Group }\end{array}$ & $\begin{array}{c}\text { Antibiotic Days } \\
\text { Control Group }\end{array}$ \\
\hline Ceftriaxone & $11(26 \%)$ & $5(12 \%)$ & 2.2 & 2 \\
\hline Ciprofloxacin/Levofloxacin & $6(14 \%)$ & $9(21 \%)$ & 2.8 & 3.9 \\
\hline Meropenem & $5(12 \%)$ & $3(7 \%)$ & 1 & 5.3 \\
\hline Nitrofurantoin & $2(5 \%)$ & $13(30 \%)$ & 3 & 4 \\
\hline Sulfamethoxazole/Trimethoprim & $2(5 \%)$ & $12(28 \%)$ & 2 & 3.2 \\
\hline Cefepime & $2(5 \%)$ & $0(0 \%)$ & 6.5 & 6 \\
\hline Doxycycline & $2(5 \%)$ & $9(21 \%)$ & 3 & 6.5 \\
\hline Tigecycline & $2(5 \%)$ & $2(5 \%)$ & 1 & 6.3 \\
\hline Ertapenem & $2(5 \%)$ & $3(7 \%)$ & 2.5 & 5.2 \\
\hline Zosyn & $0(0 \%)$ & $5(12 \%)$ & 0 & 4.2 \\
\hline Ampicillin/Amoxicillin & $1(2 \%)$ & $10(23 \%)$ & 4 & 7 \\
\hline Daptomycin & $0(0 \%)$ & $1(2 \%)$ & 0 & 1 \\
\hline Gentamicin & $1(2 \%)$ & $1(2 \%)$ & 1 & 8 \\
\hline Linezolid & $0(0 \%)$ & $3(7 \%)$ & 0 & 1.1 \\
\hline Other & $4(9 \%)$ & $7(16 \%)$ & 1.5 & 7.19 \\
\hline Average days of antibiotic therapy & & & 2.93 & 6 \\
\hline
\end{tabular}


ture positive for VRE faecium, and the other patient had ESBL Escherichia coli on a repeat urine culture.

The mean antibiotic cost per patient was lower in the fosfomycin group (\$106.74 vs. \$269.55). A reduction in cost of antibiotic therapy was seen in patients treated with fosfomycin for VRE and ESBL UTIs with cost savings of $\$ 162.81$ per patient treated. Although an overall cost savings was seen between groups, there was a higher cost associated with using fosfomycin for the treatment of non-VRE Enterococcus and non-ESBL Enterbacteriaceae UTIs (Figure 1).

\section{Discussion}

This study reiterates the clinical utility of fosfomycin against ESBL producing Enterbacteriaceae UTIs, as seen in previous studies, and establishes in vivo activity against VRE. [3, 6-13, 16-18 ]In addition, this is the first study to compare clinical outcomes of fosfomycin in the hospital setting for MDR UTIs and associated antibiotic costs. Neuner et al. conducted a retrospective chart review of 41 patients treated with fosfomcycin who had a MDR UTI from 2006 to 2010 but did not compare outcomes to other antibiotics traditionally prescribed for these pathogens. [16] UTI pathogens in the Neuner study included VRE and ESBL producers, and fosfomycin demonstrated in vitro susceptibility of $86 \%$. [16] Microbiologic cure was reported in $71 \%$ of patients with VRE and ESBL UTIs, and in our study population the cure rate was 95\%. [16] The Neuner et al. defined patient relapse as developing a UTI with the same organism within 30 days, as we did in our study. [16] In their study, $24 \%$ of patients had a relapse, whereas $5 \%$ of our patients had a UTI with the same organism within 30 days of the initial urine culture. [16] Neuner et al. concluded that fosfomycin has MDR UTI in vitro activity but that more data is needed to assess the use of fosfomycin for treating MDR UTIs. [16] The differences in clinical success and relapse rates could be due to different populations, as we evaluated all patients who received fosfomyicn, and the study conducted by Neuner and colleagues included only

Figure 1: Mean acquisition cost of antibiotic therapy per patient.

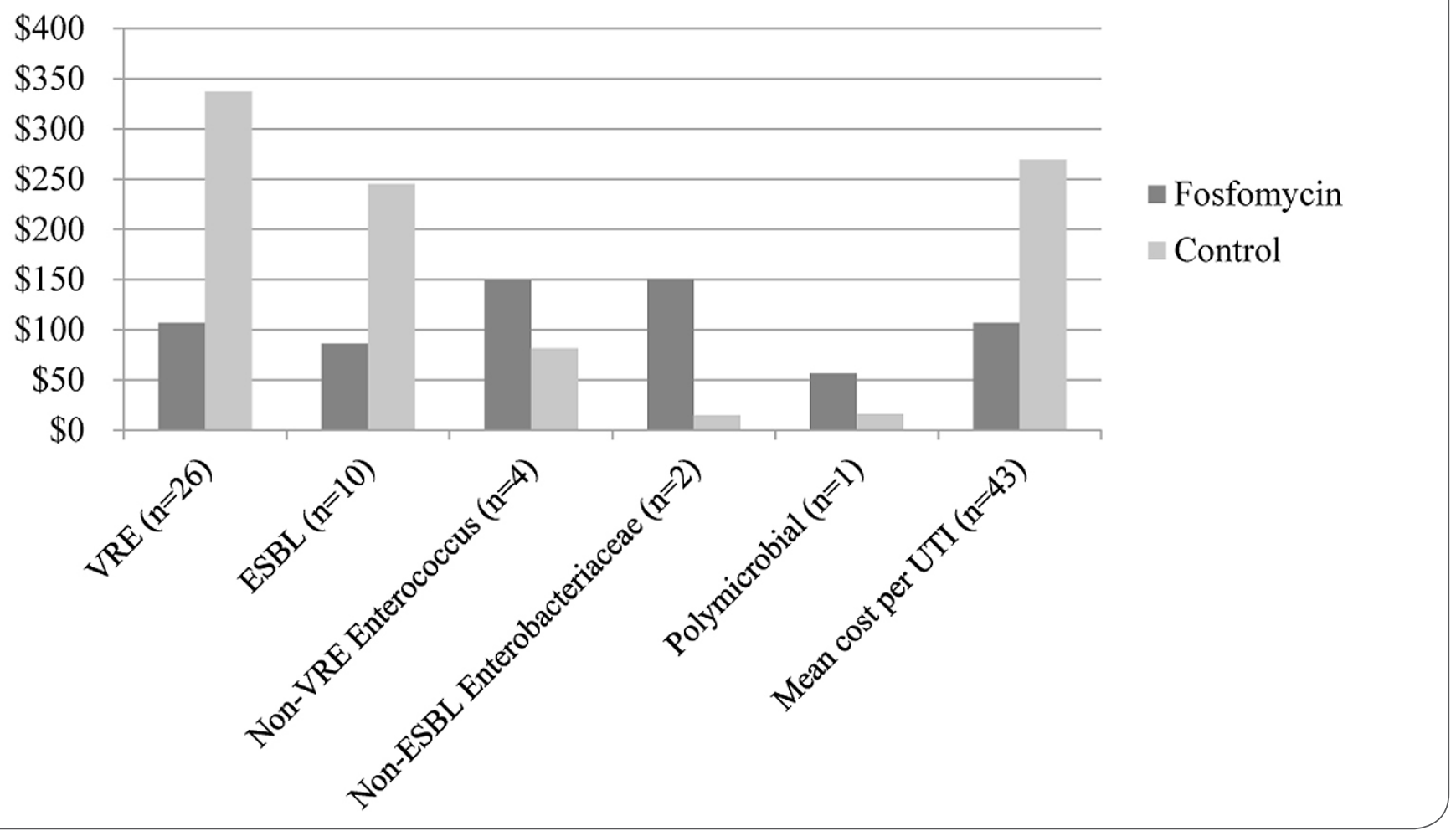


patients with MDR pathogens. Additionally, a retrospective study by Reid et al. was conducted using patient data from 2010 to 2011. [19] They specifically looked at 14 cases of gram-negative UTIs in patients who had received a kidney transplant. [19] 57\% of patients in their study were administered 3 doses of fosfomycin, whereas only $11.6 \%$ of our patients received 3 doses. [18] Their study defined recurrence as having a UTI with the same organism within 3 months of the initial diagnosis, whereas we defined clinical failure as infection with the same organism within 30 days of the original culture. [18] Reid et al. reported $54 \%$ of patients had recurrence of their UTI. [19] The difference in recurrence rates between the 3 studies is likely due to differences in sample size, UTI pathogens, inclusion population, definition of recurrence, and patient specific factors, such as immunosuppression and comorbid conditions. [19]

At the UMHS, VRE susceptibilities are routinely tested, but ESBL susceptibilities are tested only upon request. There are no official $E$. faecium breakpoints for fosfomycin, but $E$. faecalis fosfomycin breakpoints were used for both species at our institution. Our methodology focused on efficacy of fosfomycin versus other agents, and if a patient meets criteria for fosfomycin use, then we recommend fosfomycin instead of expensive antibiotics such as daptomycin or linezolid. Utilization of fosfomycin according to UMHS guideline may improve VRE and ESBL UTI treatment by shortening duration of therapy and decreasing cost while maintaining effective clinical outcomes.

Our study has several important limitations. The data was obtained from a single-center and may affect generalizability to other institutions. The retrospective study design relied on the physicians' ability to correctly distinguish between UTI and asymptomatic bacteriuria. Our ability to distinguish between asymptomatic bacteriuria and UTI was limited because symptoms attributable to a UTI were not documented in the chart for $33 \%$ of patients in the fosfomycin group and $46 \%$ of patients in the control group. However, patients presented with other potential signs of infection including leukocytosis, fever, mental status changes, or abnormal urinalysis. Thus, we've made reasonable attempts to characterize patients with UTI, with the understanding that not all patients can communicate UTIspecific symptoms, and physician documentation of UTI-specific symptoms in the medial chart is not optimal. [20]

We were also not able to demonstrate efficacy of fosfomycin alone because we analyzed efficacy for the entire antibiotic regimen from empiric to definitive therapy. Patient groups were small when treatment costs were analyzed by pathogen, and various patient factors may have skewed the cost results. A final limitation is that follow-up urine cultures to evaluate clinical efficacy were only identified if the urine culture was performed at our institution. Even with these limitations, this study supports the efficacy and cost-effectiveness of fosfomycin for treatment of lower UTI caused by VRE or ESBL producing organisms.

\section{Conclusion}

In conclusion, fosfomycin for the treatment of lower UTIs was as efficacious and in some cases more costeffective compared to other antibiotics. Treatment with fosfomycin was also associated with significant cost savings and fewer days of antibiotic therapy. Fosfomycin should be considered for the treatment of VRE and ESBL lower UTIs that are resistant to inexpensive oral antibiotics.

\section{References}

1. Heintz B, Halilovic J, Christensen C. 2010. Vancomycin-Resistant Enterococcal Urinary Tract Infections. Pharmacotherapy. 30: 1136-1149.

2. Pitout JD, Laupland KB. 2008. Extended-spectrum $\beta$-lactamaseproducing Enterobacteriaceae: an emerging public-health concern. Lancet Infect Dis. 8: 159-66.

3. Schultsz C, Geerlings S. 2012. Plasmid-mediated resistance in Enterobacteriaceae. Drugs. 72: 1-16. 
4. Swaminathan S, Alangaden G. 2010. Treatment of Resistant Enterococcal Urinary Tract Infections. Curr Infect Dis Rep. 12: 455-464.

5. Butcu M, Senbayrak Akcay S, Sengoz Inan A, Aksaray S, Ozturk Engin D, Calisici G. 2011. In vitro susceptibility of enterococci strains isolated from urine samples to fosfomycin and other antibiotics. J Infect Chemother. 17: 575-578.

6. Shrestha N, Chua J, Tuohy M, Wilson D, Procop G, Longworth D, Isada C, Hall G. 2003. Antimicrobial Susceptibility of Vancomycin-Resistant Enterococcus faecium: Potential Utility of Fosfomycin. Scand J Infect Dis. 35: 12-14.

7. Superti S, Armídio C, Dias G, Alves d'Azevedo P. 2009. In vitro Fosfomycin Activity in Vancomycin-Resistant Enterococcus faecalis. The Brazilian Journal of Infectious Diseases. 13: 123124.

8. Wilson DT and May DB. 2013. Potential role of fosfomycin in the treatment of community-acquired lower urinary tract infections caused by extended-spectrum $\beta$-lactamase-producing Escherichia coli. American Journal of Therapeutics. 20: 685-90.

9. Meier S, Weber R, Zbinden R, Ruef C, Hasse B. 2011. Extendedspectrum $\beta$-lactamase-producing Gram-negative pathogens in community-acquired urinary tract infections: an increasing challenge for antimicrobial therapy. Infection. 39: 333-340.

10. Monurol package insert. St. Louis, MO: Forest Pharmaceuticals, Inc.;2007 Jul.

11. Qiao LD, Zheng B, Chen S Yang Y, Zhang K, Guo HF, Yang B, Niu YJ, Wang Y, Shi BK, Yang WM, Zhao XK, Gao XF, Chen M. Evaluation of three-dose fosfomycin tromethamine in the treatment of patients with urinary tract infections: an uncontrolled, open-label, multicentre study. BMJ Open. 2013 Dec 4;3(12): e004157. doi: 10.1136/bmjopen-2013-004157.

12. Pullukcu H, Tasbakan M, Sipahi OR, Yamazhan T, Aydemir S, Ulusoy S. 2007. Fosfomycin in the treatment of extended spectrum beta-lactamase-producing Escherichia coli-related lower urinary tract infections. International Journal of Antimicrobial Agents. 29: 62-65.

13. Rodriguez-Baño J, Alcala JC, Cisneros JM, Grill F, Oliver A, Horcajada JP, Tortola T, Mirelis B, Navarro G, Cuenca M, Esteve M, Pena C, Llanos AC, Canton R, Pascual A. 2008. Community infections cause by extended-spectrum $\beta$-lactamase-producing Escherichia coli. Arch Intern Med. 168: 1897-1902.

14. Senol S, Tasbakan M, Pullukcu H, Sipahi OR, Sipahi H, Yamazhan T, Arda B, Ulusoy S. 2010. Carbapenem versus fosfomycin tromethanol in the treatment of extended-spectrum betalactamase-producing Escherichia coli-related complicated lower urinary tract infection. Journal of Chemotherapy. 22: 355-357.

15. Falagas ME, Kastoris AC, Kapaskelis AM, Karageorgopoulos DE. 2010. Fosfomycin for the treatment of multidrug-resistant, including extended-spectrum beta-lactamase producing, Enterobacteriaceae infections: a systematic review. Lancet Infect Dis. 10: 43-50.
16. Neuner EA, Sekeres J, Hall GS, van Duin D. 2012. Experience with fosfomycin for treatment of urinary tract infections due to multidrug-resistant organisms. Antimicrob Agents Chemother. 56: 5744-8.

17. Perri MB, Hershberger E, lonescu M, Lauter C, Zervos MJ. 2002. In vitro susceptibility of vancomycin-resistant enterococci (VRE) to fosfomycin. Diagnostic Microbiology and Infectious Disease. 42: 269-271.

18. Muvunyi $C M$, Masaisa F, Bayingana $C$, Mutesa $L$, Musemakweri A, Muhirwa G, Claeys G. 2011. Decreased susceptibility to commonly used antimicrobial agents in bacterial pathogens isolated from urinary tract infections in Rwanda: Need for new antimicrobial guidelines. American Journal of Tropical Medicine and Hygiene. 84: 923-928.

19. Reid GE, Grim SA, Layden JE, Akkina S, Tang I, Campara M, Clark NM. 2013. The Use of Fosfomycin to Treat Urinary Tract Infections in Kidney Transplant Recipients. Transplantation. 96: 12-14.

20. Hartley S, Valley S, Kuhn L, Washer LL, Gandhi T, Meddings J, Chenoweth C, Malani AN, Saint S, Srinivasan A, Flanders SA. 2013. Inappropriate Testing for Urinary Tract Infection in Hospitalized Patients: An Opportunity for Improvement Infect Control Hosp Epidemiol. 34: 1204-7.

\section{Comment on this article:}
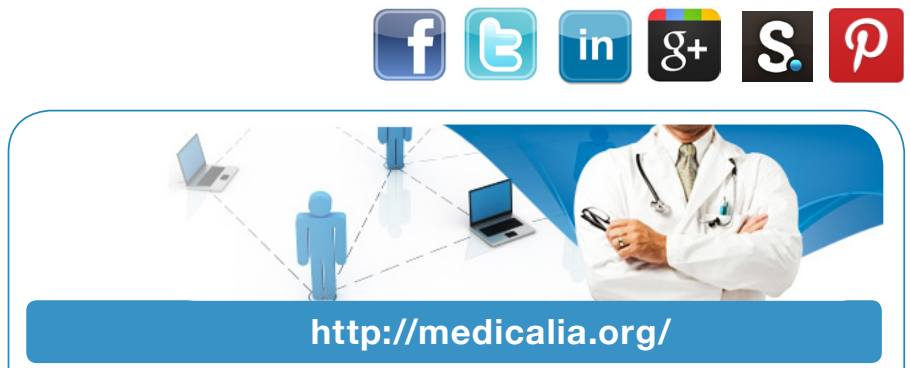

Where Doctors exchange clinical experiences, review their cases and share clinical knowledge. You can also access lots of medical publications for free. Join Now!

\section{Publish with iMedPub}

\section{http://www.imed.pub}

International Archives of Medicine is an open access journal publishing articles encompassing all aspects of medical science and clinical practice. IAM is considered a megajournal with independent sections on all areas of medicine. IAM is a really international journal with authors and board members from all around the world. The journal is widely indexed and classified Q1 in category Medicine. 